Research

\title{
Measuring health-related quality of life in patients with
} chronic obstructive pulmonary disease in a routine hospital setting: Feasibility and perceived value Preben Bendtsen*1, Matti Leijon1, Ann Sofie Sommer ${ }^{2}$ and Margareta Kristenson ${ }^{1}$

\author{
Address: ${ }^{1}$ Department of Health and Society, Linköping University, Sweden and ${ }^{2}$ Department of Pulmonary Medicine, University Hospital, \\ Linköping, Sweden \\ Email: Preben Bendtsen* - prebe@ihs.liu.se; Matti Leijon - matti.leijon@lio.se; Ann Sofie Sommer - annsofie.sommer@lio.se; \\ Margareta Kristenson - margareta.kristenson@lio.se \\ * Corresponding author
}

This article is available from: http://www.hqlo.com/content/l/I/5

(C) 2003 Bendtsen et al; licensee BioMed Central Ltd. This is an Open Access article: verbatim copying and redistribution of this article are permitted in all media for any purpose, provided this notice is preserved along with the article's original URL.

\begin{abstract}
Background: Assessment of health-related quality of life is so far mainly used in specific research settings and not widely accepted in the routine care of patients. Lack of trust in accuracy and reliability and lack of knowledge concerning the questionnaires used, methods, terminology, are just some of the perceived barriers for a more widespread dissemination of these instruments into routine health care. The present study was undertaken in order to test the feasibility of a computerised system for collecting and analysing health-related quality of life in a routine clinical setting and to examine the thoughts and attitudes among physicians concerning the value of these measurements.
\end{abstract}

Methods: Seventy-four patients with chronic pulmonary lung disease were asked to assess their health-related quality of life with a computerised version of the SF-36 questionnaire before a regular the visit to a physician. The results were immediately available for the physician during the consultation for comparison of information given by the patients and the physician's evaluation of the patients overall health status. A focus group interview with the physicians was performed before and after the implementation of routine measurements of health-related quality of life.

Results: The systematic assessment concept worked satisfactorily. All patients approached agreed to participate and completed the assessment on the touch screen computer. A weak correlation was found between patients' self-rated health and pulmonary function and between physicians' evaluation and pulmonary function. The physicians appreciated the SF-36 assessments and the value of the patients' perspective although only a few could pinpoint new clinical decisions based upon this new information.

Conclusion: Physicians' clinical evaluation and patients' self-rating of health status offer unique and important information that are complementary. 


\section{Background}

Chronic obstructive pulmonary disease (COPD) is major heath problem affecting 8-10 percent of the adult population and constitutes an important cause of death in older adults in Sweden [1]. It is thus well recognised that CODP is a source of significant disability in work life, family roles, socialisation and functions of daily living, thereby leading to decreased health-related quality of life (HRQOL) [2-7]. Previous studies have shown weak relationships between physiological variables related to CODP, such as airflow and pulmonary function, and HRQOL $[2,4,5]$. A somewhat better relationship has been seen between HRQOL and respiratory symptoms [8]. Still, patients with the same degree of airflow limitation have variable HRQOL scores, mainly due to the variability of coping strategies [7]. The goal of health services is to help patients achieve the best possible health in terms of physical and mental functioning, but also with regard to the best possible HRQOL [9]. It is therefore not surprising that HRQOL questionnaires have been introduced and used increasingly to evaluate the effects of various treatments for CODP [3]. A recent review identified 37 different questionnaires in 69 studies during the last 5 years [3]. A growing number of randomised or well-controlled studies have been performed in recent years to evaluate the effect of various drugs on respiratory diseases with regard to changes in health related quality of life [10-14]. In many of these studies the generic quality of life instrument SF36 has been used and displayed an acceptable performance [15].

However, HRQOL questionnaires are so far mainly used in specific research settings and not widely accepted in routine care for patients with COPD [3] Several barriers, on the attitudinal and knowledge level in general, have been suggested by Deyo et al., including lack of knowledge of the questionnaire instrument, methods, terminology and perceptions about subjective information [16]. The prevailing opinion appears to still be that physiological data or physicians' observations are more accurate in measuring outcome. Also, the cost of gathering, scoring and presenting questionnaire data on an individual basis has been deemed too costly or impractical in many instances $[17,18]$. However, the inclusion of the patients' perspective in outcome measurement may facilitate a more holistic approach to health care intervention since both the clinician and the patient may provide valuable information [19]. In addition, self-report not only emphasises the importance of the patients' perspective but also communicates respect to the patients with regard to their views as consumers [17].

The crucial importance of multiple perspectives in outcome assessment was emphasised in a previous study where physicians' ratings of HRQOL were only mildly re- lated to the patient ratings [17]. It was concluded from the study that incorporating patient self-report into routine care will help physicians in establishing a satisfactory relationship with their patients and improving patient satisfaction with care [17]. However, on the other hand, inclusion of HRQOL assessment as part of a routine visit might increase the patients' expectations beyond what the physician may consider necessary and feasible and thereby generate dissatisfaction when these expectations are not met [18].

In a previous study evaluating the feasibility of using the SF-36 questionnaire in a routine health care setting, the added value concerning the patients' self-perceived health status was clearly appreciated by the physicians. It was noted, however, that implementing such a system would demand a non-existent system that could immediately collect and analyse data before the patient saw the physician [20]. However, in earlier research we and others have worked on the development of computer programmes for various assessments to be used on a touch-sensitive screen and found this concept to be very user-friendly and reliable $[21,22]$. In the present study we have tested such a computerised assessment system in routine care using the SF-36 questionnaire. We hypothesised that the introduction of routine assessment of SF-36 would facilitate a learning process leading to a widening of the physicians' view upon what is important for the patients.

The present study was undertaken in order to test the feasibility of a computerised system for collecting and analysing data from the SF-36 questionnaire in a routine outpatient setting. The more specific purpose of the study was to examine the thoughts and attitudes among physicians concerning the value of an HRQOL measurement in addition to the traditional clinical and laboratory data used.

\section{Methods \\ Study design}

Patients $(\mathrm{n}=74)$ with COPD who visited the outpatient clinic at the Department of Pulmonary Medicine at the University Hospital in Linköping, in southern Sweden, were invited to assess their HRQOL using the SF-36 questionnaire. As part of an ordinary visit to the department the patients routinely performed a 1 s forced expiratory volume $\left(\mathrm{FEV}_{1}\right)$ test, immediately before seeing the physician. The study population was asked by letter to come 15 minutes before the normal scheduled time in order to perform the SF-36 test in addition to the FEV ${ }_{1}$. A computerised version of the SF-36 test was developed and a touch screen computer was set up in a relatively undisturbed area of the outpatient department. Patients, apart from a few who were very ill, were asked during a six-month period to indicate their answers by pressing directly on the 
touch screen, thereby simplifying data collection and also ensuring that all questions were answered, since the computer system demanded an answer before the next question was presented. All patients accepted and performed the required test although a few needed some minor assistance from the staff. After completing the questionnaire the computer automatically analysed and summarised the various indices and provided a printed copy of the results. The SF-36 index was then at hand for the physician in connection with the consultation.

In line with previous studies, the concept with a touch screen was seen as a highly useful way of collecting complex data and facilitating an immediate computerisation of data $[16,21]$.

Before the physician looked at the results of the SF-36 questionnaire the consultation was performed as usual. After examining the patient the physician evaluated the patient's health status on a 5-point scale from excellent to poor.

Then the physician was allowed to read the SF-36 results and thereafter indicated whether these were 1) similar to their clinical judgement, 2) added new information about the patient's health status and 3) to what extent the information from the SF-36 questionnaire was relevant for any clinical treatment decisions. All answers were to be given on a 5-point scale from "complete agreement" to "complete disagreement".

One week before this computerised collection of HRQOL was started, a focus group interview was performed with all present physicians at the department. After seven months a second focus group interview was carried out in order to evaluate changes in thoughts and attitude towards measuring HRQOL in a routine care setting. At the first interview, seven physicians were available and at the second interview nine physicians.

\section{Interview guide and data analysis}

An interview guide was developed with questions in four central themes. The first theme explored views upon the importance of evaluating the patients' perceived health and the second how the physicians usually obtain information about the patients' perspective. The third theme focused on what positive and negative consequences increased information about the patients' perceived health could have. The last theme explored feelings and thoughts about using a questionnaire when evaluating the patients' perspective.

In the second interview some specific questions were added, exploring the physicians' experience with the SF-36 questionnaire and whether the introduction had changed their encounters with the patients.

Each interview lasted for about one hour and was carried out in the library at the Department of Pulmonary Medicine. The interviews were tape-recorded and transcribed verbatim. The interviews were analysed by the second author word for word, and sorted in accordance with the four central themes.

Spearman correlations were performed, using the SPSS software, between $\mathrm{FEV}_{1}$ and the various indices of SF- 36 as well as the physician's evaluation of the patient's health status.

\section{Results \\ Correlations between SF-36 and physiological and clinical evaluations}

The correlations between FEV1 and the index of SF-36 were all non-significant (Table 1). All scales in SF-36 revealed a significant correlation with the physicians' ratings of the patients' general health status on a 5-point scale. The highest correlation was seen between the physical functioning scale of the SF-36 ( $\mathrm{r}=0.55)$ and the lowest with the pain and emotional role scale $(\mathrm{r}=0.29$ for both scales) (Table 1). The remaining scales of SF-36 and the physicians' ratings revealed correlations somewhere in between these two levels. The correlation between the physicians' ratings and FEV1 was not significant.

In Table 2 the physicians' evaluation of the value of SF-36 measurement to their clinical evaluation is displayed. In all, 9 percent of the physicians indicated that their clinical evaluation completely agreed with the patients' evaluation and a further $63 \%$ agreed to some extent. Fourteen percent agreed to some extent that new information not

Table I: Correlations between the SF-36 index, FEVI and the physicians' ratings of the patients' health status.

\begin{tabular}{lll}
\hline SF-36 index & FEV & Physicians' rating I) \\
\hline Physical functioning & $0.19 \mathrm{NS}$ & $0.55 * * *$ \\
Physical role & $0.06 \mathrm{NS}$ & $0.40 * *$ \\
Pain & $-0.19 \mathrm{NS}$ & $0.29 *$ \\
General health & $-0.0 \mathrm{I} \mathrm{NS}$ & $0.36 * *$ \\
Vitality & $-0.06 \mathrm{NS}$ & $0.35 * *$ \\
Social functioning & $0.02 \mathrm{NS}$ & $0.40 * *$ \\
Emotional role & $0.10 \mathrm{NS}$ & $0.29 *$ \\
Mental health & $0.10 \mathrm{NS}$ & $0.40 * *$ \\
FEV & 1.00 & $0.18 \mathrm{NS}$ \\
Physician's rating & $0.18 \mathrm{NS}$ & 1.00
\end{tabular}

NS $=$ Non significant, $*=P<0.05, * * p<0.01, * * * p<0.00$ I I) Single question evaluation of the patients' general health on a 5 -point scale from excellent to poor. 
Table 2: Physicians' evaluation of the correlation between SF-36 and their clinical judgement of the patients' general health status.

\begin{tabular}{|c|c|c|c|c|c|}
\hline & Agree completely & $\begin{array}{l}\text { Agree to some } \\
\text { extent }\end{array}$ & $\begin{array}{l}\text { Neither agree } \\
\text { nor disagree }\end{array}$ & Disagree & Disagree completely \\
\hline & \multicolumn{5}{|c|}{ Percentage of affirmative answers } \\
\hline $\begin{array}{l}\text { I. The SF-36 index correlates well with my clinical } \\
\text { judgement }\end{array}$ & 9 & 63 & 25 & 3 & 0 \\
\hline 2. The SF-36 gave new information not known earlier & 0 & 14 & 53 & 21 & 12 \\
\hline $\begin{array}{l}\text { 3. The SF- } 36 \text { gave new information relevant to the clinical } \\
\text { decision }\end{array}$ & 0 & 8 & 24 & 42 & 26 \\
\hline
\end{tabular}

known earlier was revealed by the SF-36 questionnaire. A total of $53 \%$ were indecisive as to whether new information was uncovered. In 8 percent of the consultations this new information had some clinical implication according to the physicians.

The staff supervising the patients during the assessment did not observe any disapproval and most patients expressed a positive view upon the assessment.

\section{First interview with the physicians performed before the routine assessment of HRQOL}

Views on the holistic approach

The physicians were aware that pulmonary function tests have a low correlation with the patients' subjective health status. One physician stated: "We do not believe that pulmonary function tests give us a true picture - instead they give us very little information". Concerning the value of HRQOL measurements the physicians welcomed a broader assessment of the patients' health status than is normally the case. This was considered particularly important in a chronic disease such as COPD. There was a consensus that the content of medical education influences the physicians' frame of reference and the focus physicians have upon patients' health status. One physician stated: "I believe that since the medical education focuses on medical data instead of the patients' perspective, physicians are not conscious about the patients' perspective to the same extent".

Views on the value of outcome assessments in patients with COPD The physicians expressed a concern for the present methods of evaluating patients with COPD. The routine data such as $\mathrm{FEV}_{1}$ were not recognised as reliable but thought to only offer a small amount of information. The physicians welcomed more valid methods such as HRQOL for evaluating the effects of new drugs as one example.

"As it is now we do not evaluate the effect of new drugs and a method for this would be very much appreciated."
Consequence of outcome assessments of health care interventions The participants emphasised that the patient's own evaluation broadens the understanding for what kind of health care interventions the patients need. There was a consensus about the important role of other staff members such as physiotherapists and the participants expressed a concern about the amount of drugs prescribed to patients with COPD.

"Some patients might be better off having regular contact with staff members other than the physicians."

One physician stated that perhaps many patients with COPD have too much medication and systematic patientcentred clinical evaluations might prove this.

"If we could receive more valid information we might be able to withdraw some of the drugs that is not necessary."

There was agreement that another consequence of introducing a systematic patient-focused evaluation of health care interventions could be a more effective use of allocated resources.

"This could lead to a reallocation of funds from drug treatments to other forms of treatment, offered by a physiotherapist as one example."

\section{Expected value of SF-36 measurement as part of the daily routine} care

The participants agreed that by introducing HRQOL as part of the daily routine in the outpatient department there could be a change in the focus of the physicians' questions to the patients as part of the examination. One physician stated:

"You might discover new dimensions of the patients' wellbeing."

Furthermore, the physicians expect the patients to react positively when they ask about subjective health parameters, as one physician expressed it: 
"Some patients will become healthier simply as a consequence of you asking about more subjective health matters."

However, a concern was expressed that certain patients might not give honest answers to the SF-36 questions due to fear of discontinuation of treatment. Another fear was expressed concerning the impact of other diseases upon HRQOL. The patients might improve in his/her COPD but deteriorate in another disease, leading to a complex interpretation of the HRQOL questionnaire.

"You can apply the SF-36 in a group of patients as well as in an individual patient as long as you are more cautious with the interpretation of the results in an individual patient. "

With regard to how to use the HRQOL index in communication with the patients one physician stated:

"The question is whether one should use an HRQOL questionnaire as part of the consultation or more as an instrument in order to teach the physicians to have a more holistic view upon disease and the impact upon the patients lives. "

Second interview after the implementing of the routine assessment of HRQOL

Views on a holistic approach

As seen in the first interview, there was a consensus that a holistic approach including HRQOL assessments is necessary when treating patients, and not least for patients with COPD. It was agreed that a holistic approach could facilitate the patients' satisfaction with the health care intervention since the physician could discuss the expectations from the patients before the treatment.

"In some instances the treatment aims at minimising a deterioration in the patient's health status rather than improving the health."

Some physicians have learned, during the project time that patients with an apparently similar medical condition assessed their HRQOL very differently. The physicians stated that various personal and social resources might affect the experienced HRQOL:

"The degree of impairment in HRQOL depends partly upon the surroundings; whether the patients have children, a spouse or friends. These are dimensions that medication cannot affect."

Views on the value of outcome assessments of patients with COPD The physicians agreed that evaluation of treatment progress before the introduction of HRQOL assessment have lacked the patients' perspective. The change of focus was in line with the general trend in the health care sector. One physician stated:
"The measurement of HRQOL is popular these days, you cannot do a study without including this one way or another."

The physicians agreed that most previous evaluations have focussed on physiological "objective" parameters and they appreciated the more subjective patient-focused measurements like the SF-36

"We are searching for a method that can give us a more comprehensive description of the effect of a certain treatment..."

The concept of a computerised assessment system with immediate feedback to the physician and the patients was appreciated as very comprehensive.

"The concept with a touch screen is excellent and makes the assessment possible in a routine care setting. "

\section{Consequence of outcome assessments of health care interventions} Although the physicians were presented with HRQOL assessment in the individual patient's consultation they requested more data on a group level in order to be able to compare different treatment approaches and patient groups. One physician explained why they wanted data on a group level:

"As part of the quality control in order to be able to show what we are doing."

The introduction of HRQOL measurement in routine care has encouraged one physician to think about the prescription of drugs in patients without any change in the HRQOL. He stated:

"The physician lacks the courage to say to the patient that all resources have been used and instead he adds more drugs to the patient's treatment."

The participants all agreed that they wanted to continue with HRQOL measurement as part of their daily routine care and make further adjustment in the routine and handling of HRQOL measurements.

Perceived value of SF-36 measurement in the daily routine care One physician explained how the SF-36 index has helped him in starting a discussion with the patient:

"How come that you experience this and this..."

There was a consensus that HRQOL measurements would be good to have on a group level in order to compare different treatment approaches. Although some physicians stated that the SF-36 questionnaire did not reveal new clinical information they still valuated the self-report by the patients. One physician stated: 
"I believe that the SF-36 measurements I have seen only confirm the picture I had of the patients already. This does not indicate that the measurement is not useful and perhaps with further experience in the field it will show to be valuable."

\section{Discussion}

The study explored the feasibility of a routine collection of HRQOL information in an outpatient setting. A generic HRQOL questionnaire, the SF-36, was chosen instead of a disease specific in order to examine the added value of a broader HRQOL assessment in patients with COPD. As a consequence of this all correlations performed with more disease specific parameters would probably had been stronger if a specific HRQOL questionnaire had been chosen. Still a generic questionnaire was considered appropriate for widening the physician's view upon what is important for the patients concerning the overall health related quality of life due to COPD as well as other health conditions.

As expected from earlier reports the physician's evaluation of the patient's health status correlated better with the patient's perception of HRQOL than the physiologic measures (Table 1) [4]. Although varying from 0.29 to 0.55 all correlations between SF-36 and the physician's overall assessment were significantly correlated. Not surprisingly the physicians seem to be better at detecting physical limitations than mental and social limitations. The added value of applying measurement which includes the patient's perceived mental and social status is obviously needed in order to get a holistic perspective of the patient's situation [17]. Thus, both clinician and self-report ratings provide valuable information in order to understand the total impact of disease on the patient's life.

Most physicians indicated that SF-36 measurement did not give new information not known earlier and only $8 \%$ indicated that the information from the SF-36 gave rise to new clinical decisions (Table 2). However, the SF-36 evaluation might still add valuable information for the physician's understanding of the disease impact upon the patient although this might not directly lead to new clinical decisions. This was also confirmed in the focus group interviews. This finding thus extends a previous report where physicians appreciated the potential value of having access to SF-36 results during a consultation [20].

From both our focus group interviews it is apparent that the physicians agree that the health care system is far too focused on traditional medical parameters. Previously the department's focus has been on the disease, such as signs and symptoms instead of self-perceived indicators of health. Although the physicians had been aware of the poor correlation between physiological parameters and experienced health, they still had not found another way of assessing treatment progress. There was agreement that HRQOL assessment is one way of introducing a broader perspective, although some physicians appear to prefer to learn to ask these kinds of questions themselves as part of a normal consultation. The participants agreed that there is an additional value in a routine collection of standardised HRQOL measurement such as the SF-36 with regard to data on a group level as reported in another study by Ruta et al. [20]. In addition to the added value of individual patients' assessments, the physicians in our study also wanted to be able to show the effect of various treatment approaches on a group level. This is probably a consequence of long-term work with Total Quality Management (TQM) in this department.

By introducing HRQOL assessment as part of the daily routine the physician have started to think more about the total disease impact on the patient's life situation. The HRQOL has initiated a process of learning and extended the ongoing quality assurance process in the organisation. Thus, the participants welcome on the one hand a more holistic approach to the management of the patients as offered by the SF-36, but when it comes to individual discussion with the patients, some physicians appear hesitant concerning the added value of SF-36. One possible reason for this could be a perceived difficulty or lack of experiences in talking about psychosocial matters and, more importantly, how to give competent advice. It appears too easy to write another prescription rather than prescribe "psychosocial action". In a critical assessment of quality of life studies, McCarthy suggests that the resistance from physicians towards measurements of quality of life is that they do not want to uncover too many problems [18]. Another reason why the physicians did not fully appreciate the added information from the patient's assessment could be a natural inexperience with a new method.

Concerning whether the routine assessment of SF-36 would add new information about the patient's perceived limitations, the physicians expressed a hope about this before the start of the project. After the implementation of the project there were diverging opinions about the information gained from the assessment. Some expressed that the SF-36 index helped them to ask some specific questions to the patients, whereas others only thought that the assessment confirmed the picture they had before, i.e. that they already included questions about the patient's view of the disease impact. Another possible explanation could be that the physicians simply did not consider the value of the information that was included in the patient self-report. In fact this could be a common misunderstanding that could be an important barrier for a routine collection of patient self-report of outcome. As stated before, both the clinician and the patient self-report are important 
sources of information and should not be considered as in opposition to each other [17].

\section{Conclusion}

The aim of this study was to study the feasibility and perceived value of a routine assessment of HRQOL in a group of patients with COPD in an outpatient department. The concept included an immediate HRQOL assessment made by the patients 15 minutes before the actual consultation with the physician. Thereby the physician could discuss the SF-36 index directly with the patients. The systematic assessment concept worked satisfactorily. All patients approached agreed to participate and were able to complete the assessment on the touch screen computer. A weak correlation was found between patients' self-rated health and pulmonary function and between physicians' evaluation and pulmonary function. These correlations would probably have been stronger if a disease specific questionnaire was chosen instead of the generic SF-36 questionnaire. However, the physicians appreciated the SF-36 assessments and the value of the patient's perspective although only a few could pinpoint new clinical decisions based upon this new information. All physicians agreed that the information is very valuable on a group level in order to compare various treatment approaches. There was a consensus on the need for a more holistic approach in the health care sector, and introducing a patient-centred outcome assessment such as the SF-36 could start a learning process in the organisation towards this goal. It is important to emphasise that the clinical evaluation and the patient's self-rating both offer unique and important information. These perspectives are both needed and they complement each other.

\section{Authors' Contributions}

PB participated in the design of the study and the analysis of the results and revising the manuscript

ML participated tin the design of the study carried out the focus group interview and did the first analysis and draft of the paper.

AS participated in the design of the study and coordinated the study at the clinic and participated in the analysis of the data and revising the manuscript

MK participated in the design of the study and the analysis of the results and revising the manuscript

All authors read and approved the final manuscript.

\section{Acknowledgements}

We would like to thank the staff as well as the patients at the Department of Pulmonary Medicine at the University hospital in Linköping, Sweden for their participation in the project.
A special thanks to Marianne Rimås for her interest in measuring quality of life in the patients with COPD and the management of the department for giving a high priority to HRQOL measurements at the department.

\section{References}

I. Socialstyrelsen Sjukvården i Sverige 1998 In: Book Sjukvården $i$ Sverige City: Socialstyrelsen 1998 ,

2. Tsukino M, Nishimura K, Ikeda A, Koyama H, Mishima M and Izumi T Physiologic factors that determine the health-related quality of life in patients with COPD Chest 1996, I I 0:896-903

3. Cullen $D L$ Measures of functional status and quality of life in chronic obstructive pulmonary disease Monaldi Archives of Chest Diseases 1999, 54: 183-5

4. Selim AJ, Ren XS, Fincke G, Rogers W, Lee A and Kazis L A symptom-based measure of the severity of chronic lung disease Chest 1997, I I I:1607-14

5. Guyati GH, Townsend M, Berman LB and Pugsley SO Quality of life in patients with chronic airflow limitations British Journal of Diseases of the Chest 1987, 81:45-54

6. Curtis JR, Deyo A and Hudson LD Health-related quality of life among patients with chronic obstructive pulmonary disease Thorax 1994, 49: 162-70

7. Herbert $R$ and Gregor $F$ Quality of life and coping strategies of clients with COPD Rehabilitation Nursing 1997, 22:182-7

8. Hajiro T, Nishimura K, Jones PW, Tsukino M, Ikeda A, Kpyama $\mathrm{H}$ and Izumi T A novel, short, and simple Questionaire to measure health-related quality of life in patients with chronic obstructive pulmonary disease American Journal of Respiratory Critical Care Med 1999, 159:1874-78

9. WHO Health $2 I$ - health for all in the $2 I$ st century Copenhagen: World Health Organisation 1999 ,

10. Hyland ME and Crocker GR Validation of an asthma quality of life diary in a clinical trial Thorax 1995, 50:724-30

II. Mahajan P, Okamoto LJ, Schaberg A, Kellerman D and Schoenwetter WF Impact of fluticasone propionate powder on health-related quality of life in patients with stable asthma Journal of Asthma 1997, 34:227-34

12. Mahler DA, Faryniarz K, Tomlison D, Cilice G, Robins AG, Olmstead

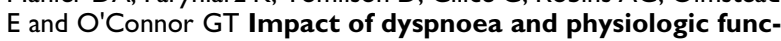
tions on general health status in patients with chronic pulmonary disease Chest 1992, 102:395-40I

13. MaFadden ER, Casale TB, Kemp JP, Metzger WJ and Nelson HS Administration of bedesonide once daily by means of turbuhaler to subjects with stable asthma Journal of Allergy and Clinical Immunology 1999, 104:46-52

14. Noonan M, Chervinsky P, Busse WW, Weisberg SC, Pinnas J and de Boisblanc BP Flutiacasone prioprionate reduces oral prednisone use while it improves asthma control and quality of life American Journal of Respiratory Critical Care Medicine 1995, I52:1467-73

15. Ware JE, Kemp JP, Buchner DA, Singer AE, Nolop KB and Goss TF The responsiveness of disease-specific and generic health measures to change in the severity of asthma among adults Quality of Life Research 1998, 7:235-44

16. Deyo RA and Patrick DL Barriers to the use of health status measures in clinical investigations, patient care and policy research Medical Care 1989, 27:254-68

17. Russo J, Roy-Byrne P, Jaffe C, Ries R, Dagakadis C, Dwyer-O'Connor $E$ and Reeder $D$ The relationship between patient-administered outcome assessments to quality of life and physician ratings: Validity of the BASIS-32 The Journal of Mental Health Administration 1997, 24:200-14

18. McCarthy DM Quality of Life: A critical assessment Scandinavian Journal of Gastroenterology 1995, 30:141-6

19. Vang J Health outcomes and evaluations: context, concepts and successful applications Leeds: European Clearing Houses on Health Outcomes 1997,

20. Ruta D, Coutts A, Abdalla M, Masson J, Russell E, brunt P, McKinlay $A$, Mowat $A$ and Sinclair $T$ Feasibility of monitoring patient based health outcomes in a routine hospital setting Quality in Health Care 1995, 4:161-5

21. Bendtsen P and Timpka T Acceptability of computerized self-report of alcohol habits: a patient perspective Alcohol Alcohol 1999, 34:575-80 
22. Buxton J, White $M$ and Osoba D Patients' experiences using a computerized program with a touch-sensitive video monitor for the assessment of health-related quality of life Quality of Life Research 1998, 7:513-9

Publish with Bio Med Central and every scientist can read your work free of charge

"BioMed Central will be the most significant development for disseminating the results of biomedical research in our lifetime. " Sir Paul Nurse, Cancer Research UK

Your research papers will be:

- available free of charge to the entire biomedical community

- peer reviewed and published immediately upon acceptance

- cited in PubMed and archived on PubMed Central

- yours - you keep the copyright

Submit your manuscript here:

http://www.biomedcentral.com/info/publishing_adv.asp 